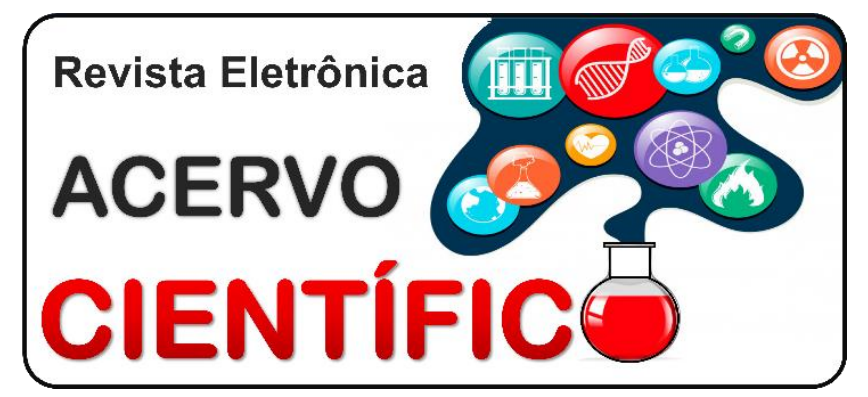

ARTIGO ORIGINAL

Recebido em: 6/2020

Aceito em: $7 / 2020$

Publicado em: 1/2021

\title{
Prevalência de sobrepeso e obesidade em escola pública de uma cidade do Pará
}

\author{
Prevalence of overweight and obesity in a public school in a city in Pará \\ Prevalencia de sobrepeso y obesidade en una escuela pública de una ciudad de Pará \\ Karolyne Bezerra de Oliveira1*, Thayná Silva Cotrim¹, James Santos Aguiar ${ }^{1}$, Patrícia Reis \\ Valente $^{1}$, Ana Paula Ramos de Souza ${ }^{1}$, Murilo Brandão Pimenta ${ }^{1}$, Darlinton Cardoso Fonseca ${ }^{1}$, \\ Vinicius Francisco Fernandes Rodrigues ${ }^{1}$, Yanka Rafaela da Costa Neto Vieira ${ }^{1}$, Cláudia Nazaré \\ de Souza Almeida Titan Martins ${ }^{1}$.
}

Resumo: O objetivo do estudo é verificar a prevalência de sobrepeso e obesidade em adolescentes matriculados no sexto ano letivo de uma escola pública de uma cidade do Pará. Estudo transversal de caráter observacional, quantitativo do tipo descritivo e analítico, foram utilizados para a pesquisa todos os adolescentes que se enquadram nos critérios de inclusão. Foi utilizado de balanças e fitas métricas para aferição dos dados necessários para a pesquisa, ao mesmo tempo em que questionados o sexo e a idade desses jovens para que se possa comparar as diferenças entre os sexos e que se possa determinar o grupo etário em que cada um se encaixou. Foram analisados 62 adolescentes, sendo 28 do sexo feminino e o restando do masculino. A média de idade foi 12 anos, $69,4 \%$ foi classificado como eutrófico, nota-se proporções semelhante de adolescentes com altura entre 1,0 - 1,49 $\mathrm{m}(48,4 \%)$ e entre 1,5 - 1,99 $(51,6 \%)$, maior frequência entre 60 e $69 \mathrm{~cm}(42,0 \%)$ de circunferência abdominal. A avaliação dos resultados mostrou que a prevalência de sobrepeso entre o grupo feminino é maior que o masculino.

Palavras-chave: Epidemiologia, Obesidade, Adolescentes.

\begin{abstract}
The objective of the study is to verify the prevalence of overweight and obesity in adolescents enrolled in the sixth academic year of a public school in a city in Pará. An observational, quantitative, descriptive and analytical cross-sectional study, all adolescents were used for the research. that fit the inclusion criteria. Scales and measuring tapes were used to measure the data needed for the research, while questioning the gender and age of these young people so that the differences between the sexes can be compared and the age group in which each can be determined. one fit. 62 adolescents were analyzed, 28 of whom were female and the remainder were male. The average age was 12 years old, $69.4 \%$ was classified as eutrophic, similar proportions of adolescents with height between $1.0-1.49 \mathrm{~m}(48.4 \%)$ and between 1.5 $1.99(51.6 \%)$, higher frequency between 60 and $69 \mathrm{~cm}(42.0 \%)$ of waist circumference. The evaluation of the results showed that the prevalence of overweight among the female group is higher than the male.
\end{abstract}

Keywords: Epidemiology, Obesity, Adolescent.

Resumen: El objetivo del estudio es verificar la prevalencia de sobrepeso y obesidad en adolescentes matriculados en el sexto año académico de una escuela pública en una ciudad de Pará. En un estudio transversal observacional, cuantitativo, descriptivo y analítico, se utilizaron todos los adolescentes para la investigación. que se ajustan a los criterios de inclusión. Se utilizaron escalas y cintas de medición para

${ }^{1}$ Centro Universitário Metropolitano da Amazônia (UNIFAMAZ), Belém-Pará. *E-mail: karolyne.deoliveira@icloud.com 
medir los datos necesarios para la investigación, al tiempo que se cuestionaba el género y la edad de estos jóvenes para poder comparar las diferencias entre los sexos y el grupo de edad en el que se pueden determinar. uno en forma. Se analizaron 62 adolescentes, 28 de los cuales eran mujeres y el resto hombres. La edad promedio fue de 12 años, el $69.4 \%$ fue clasificado como eutrófico, proporciones similares de adolescentes con altura entre $1.0-1.49 \mathrm{~m}(48.4 \%)$ y entre $1.5-1.99(51,6 \%)$, mayor frecuencia entre 60 y $69 \mathrm{~cm}(42,0 \%)$ de circunferencia de la cintura. La evaluación de los resultados mostró que la prevalencia de sobrepeso entre el grupo femenino es mayor que la masculina.

Palabras clave: Epidemiologia, Obesidad, Adolescente.

\section{INTRODUÇÃO}

A globalização, associada a enorme velocidade da informação, padronizam modos de vida com mudanças semelhantes em todo o mundo, mostrando um padrão alimentar inadequado aliado a uma inatividade física cada vez maior. O Brasil, assim como outros países em desenvolvimento, passa por período de transição epidemiológica, caracterizada por uma mudança no perfil dos problemas relacionados à saúde pública, predominando as doenças crônico-degenerativas, embora as doenças transmissíveis ainda desempenhem papel importante. Esta transição acompanha-se de modificações demográficas e nutricionais, com a desnutrição sendo reduzida a índices cada vez menores e a obesidade atingindo proporções epidêmicas (SBP, 2012).

A Obesidade pode ser definida como uma síndrome metabólica caracterizada como complexa, de difícil controle, refletida por acúmulo excessivo de gordura que excede aos padrões estruturais e físicos do corpo, e que apresenta graves dimensões sociais e psicológicas, principalmente entre as crianças que estão iniciando sua vida social e que por causa da obesidade podem sofrer preconceitos além de que estão desenvolvendo seu corpo e dessa forma podem gerar desequilíbrios hormonais que perpetuarão para o resto da vida (JUNIO JGS, 2012). Embora os genes desempenhem papel importante na programação dos potentes mecanismos fisiologicos que regulam a ingestão alimentar e o metabolismo energético, o estilo de vida e os fatores ambientais podem representar papel dominante em muitas pessoas obesas (GUYTON AC, et al., 2011). Dessa forma, é de extrema importância o desenvolvimento de estudos e pesquisas nesta área a fim de prevenir que jovens e adolescentes entrem na obesidade, pois dessa forma gerará maior número de pessoas com comorbidades (Hipertensão Arterial Sistêmica, Diabetes Mellitus, Infarto Agudo do Miocárdio por exemplo).

A Organização Mundial de Saúde aponta a obesidade como um dos maiores problemas de saúde pública no mundo. A projeção é que, em 2025, cerca de 2,3 bilhões de adultos estejam com sobrepeso; e mais de 700 milhões, obesos. O número de crianças com sobrepeso e obesidade no mundo poderia chegar a 75 milhões, caso nada seja feito (BRASIL, 2010).

O número de crianças e adolescentes com sobrepeso e obesidade, no mundo, tem aumentado dramaticamente no decorrer nos últimos 20 anos por diversos fatores. Fatores econômicos e sociais tais como o aumento da economia familiar deveria propiciar à alimentação equilibrada e saudável, contudo ocorre o oposto: a inserção de crianças cada vez mais cedo na alimentação de fast-food, a base de enalatados, gorduras saturadas/insaturadas e com altos níveis de sódio e carboidratos aliado à falta de estímulo à atividade física gera um complexo sistema de criação de doenças cardiovasculares tais como a hipertensão arterial sistêmica, insensibilidade à insulina gerando Diabetes Mellitus e, por fim, a obesidade (BRASIL, 2010).

$\mathrm{Na}$ faixa etária pediátrica, estudos nacionais demonstram prevalências de excesso de peso que variam entre $10,8 \%$ a 33,8\% em diferentes regiões (BRASIL LMP, et al., 2007). Todas as fases da infância são importantes para o estudo da obesidade, porém, a adolescência representa um período crítico para o desenvolvimento do excesso de peso. Considera-se que o risco de um adolescente obeso manter-se assim até a idade adulta chega a aproximadamente $80 \%$ (CAMPOS LA, et al., 2007).

Em geral, não é difícil reconhecer a obesidade ou até mesmo o sobrepeso, mas o diagnóstico correto requer que se identifiquem os níveis de risco, o que, frequentemente, necessita de algumas formas de 
quantificação. Recentemente, técnicas de imagem, tais como ressonância magnética, tomografia computadorizada e absorciometria com raios- $X$ de dupla energia, têm sido alternativas, mas o custo e a falta dos equipamentos necessários impedem o uso dessas técnicas na prática clínica (ABESO, 2009).

O uso de indicadores antropométricos na avaliação do estado nutricional de indivíduos ou coletividades é, entre várias opções, a mais adequada e viável para ser adotada em serviços de saúde, considerando suas vantagens como: baixo custo, a simplicidade de realização, sua facilidade de aplicação e padronização, amplitude dos aspectos analisados, além de não ser invasiva (BRASIL, 2011).

Embora existam vários indicadores para definir sobrepeso e obesidade, o índice de massa corporal (IMC) tem sido recomendado pela Organização Mundial da Saúde (OMS) para avaliação do estado nutricional (DUMITH SC, et al., 2010). Para adolescentes, isto é, indivíduos com idade entre maior ou igual a 10 anos e menor de 20 anos, é realizada a partir da identificação do percentil de IMC por idade. $O$ valor obtido de IMC e a idade do adolescente devem ser identificados no gráfico de IMC por idade, segundo o sexo do indivíduo. O percentil de IMC por idade em que se encontra o adolescente também pode ser identificado por meio de tabelas que apresentam diferentes valores de IMC em função da idade e do sexo do indivíduo (BRASIL, 2010).

Diante do exposto, conclui-se que é necessário identificar precocemente distúrbios nutricionais e intervir contra suas possíveis consequências. Logo, o objetivo do estudo é analisar uma população de adolescentes estudantes de uma escola do Pará em busca de taxas de sobrepeso e obesidade.

\section{MÉTODOS}

Trata-se de um estudo transversal de caráter observacional, quantitativo do tipo descritivo e analítico. O projeto foi aprovado pelo Comitê de Ética em Pesquisa sob número de parecer 1.832.881. Dessa maneira, busca-se obedecer a resolução 466 do Conselho Nacional de Saúde (CNS).

O estudo protegeu os participantes da pesquisa científica envolvendo seres humanos e considerou o respeito a dignidade humana como primordial e necessário para a pesquisa. Além disso, assegurou o uso de dados de forma confidencial, utilizando-os apenas para fins do projeto. Garantiu-se também, o comprometimento dos pesquisadores em expor de maneira sucinta os resultados alcançados sem restrições.

A população alvo da pesquisa era formada por alunos de ambos os sexos, regularmente matriculados nas turmas do sexto ano letivo de uma Escola Pública Estadual de uma cidade do Pará, composta por aproximadamente 96 discentes com idades entre 10 e 19 anos. Considerando esta População de adolescentes e um erro máximo de estimativa de 5\%, obteve-se um tamanho amostral mínimo de 77 alunos que seriam aleatoriamente selecionados. No entanto, por motivos relativos à gestão da referida Escola, só foi possível desenvolver o estudo com 62 adolescentes, caracterizando assim amostragem por conveniência, porém sem prejuízo da Normalidade, visto que, para $n>30$, a distribuição amostral das médias é aproximadamente Normal.

As medidas antropométricas (massa corporal e estatura) foram coletadas nas dependências da escola durante os intervalos das aulas, observando a orientação de que os alunos não tivessem realizado quaisquer tipos de atividades físicas antes da avaliação. Foi utilizada uma balança portátil digital G-TECH e uma fita métrica, ambas previamente calibradas.

Durante as aferições, executadas pelos pesquisadores, os adolescentes permaneceram vestidos com roupas leves, sem calçados e/ou quaisquer tipos de adereços, como bonés, relógios, etc. Para aferir a massa corporal, o participante permaneceu em posição ortostática, de forma que o peso do corpo ficasse igualmente distribuído. Para aferir a estatura, o aluno foi posicionado na posição ortostática e com os pés unidos, procurando pôr em contato com o instrumento de medida as superfícies posteriores do calcanhar, cintura pélvica, cintura escapular e região occipital. A medida foi feita com o aluno em apneia inspiratória, de modo a minimizar possíveis variações sobre esta variável antropométrica. A fita métrica se estendeu do ponto mais baixo do calcanhar até a altura do vértex (ponto mais alto da cabeça). 
Foram excluídos os adolescentes que não apresentaram o Termo de Consentimento Livre e Esclarecido (TCLE) previamente assinado pelos pais ou responsáveis e/ou que não estivessem adequadamente vestidos para a tomada das medidas antropométricas.

Os dados referentes às características sociodemográficas e clínicas foram tratados utilizando estatística descritiva, expressos sob a forma de Média \pm Desvio Padrão, Mediana e Percentis e frequências absoluta e relativa, conforme o caso, e apresentados em tabelas e/ou gráficos.

A associação entre variáveis categóricas foi avaliada utilizando o teste do Qui-Quadrado (ou teste G, Exato de Fisher ou Razão de Chances, conforme a necessidade) e, no caso de variáveis quantitativas, a diferença entre as médias foi testado pelo teste $t$ de Student. Todos os testes foram executados com o auxílio do software BioEstat 5.4 (AYRES M, et al., 2008), e resultados com $p \leq 0.05$ (bilateral) foram considerados significativos.

\section{RESULTADOS}

O presente estudo permitiu classificar o perfil nutricional de adolescentes de uma Escola Pública Estadual inserida na realidade da Unidade Básica de Saúde do Benguí II. Participaram da análise 62 adolescentes, desses, 28 eram meninas e 34 meninos, ou seja, uma proporção semelhante entres os gêneros $(p=0,5254)$. Os avaliados possuíam entre 11 a 15 anos, com maior frequência de alunos com 12 anos de idade $(51,6 \%)$ e menor frequência com 14 e 15 anos, correspondendo respectivamente a $1,6 \%$ e $3,2 \%(p<0,0001)$. Os adolescentes foram classificados de acordo com o escore-z da OMS (2007), $p<0,0001$. A maioria apresentou-se eutrófico $69,4 \%$, que representa 43 indivíduos. Entretanto, mesmo em menor frequência, foi possível detectar alterações como magreza (1) $1,6 \%$ (escore $Z<-2$ ), risco de sobrepeso (1) $1,6 \%$ e obesidade grave (1) $1,6 \%$ (escore $Z>+3$ ), além de $16,1 \%$ estarem já na categoria de sobrepeso e $9,7 \%$ em obesidade (Figura 1).

Figura 1 - Avaliação do estado nutricional segundo a OMS.

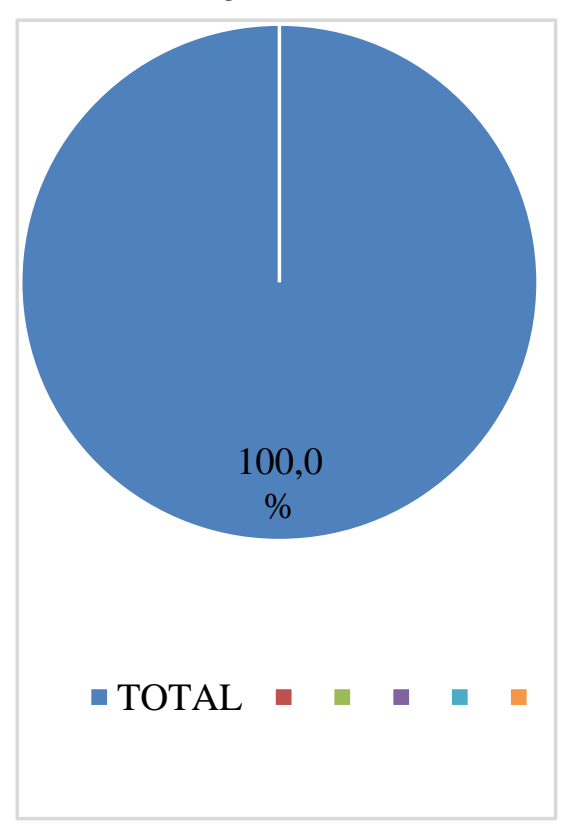

Fonte: Oliveira KBO, et al., 2020.

Quando analisados por sexo e faixa etária, o grupo masculino, de 11 a 15 anos, apresentou 3\% (1) magreza, $71 \%$ (24) eutrofia, 3\% (1) risco de sobrepeso, 12\% (4) sobrepeso, 12\% (4) obesidade e nenhum caso com obesidade grave. Na classificação feminina de 11 a 15 anos a figura mostra que, não houveram casos de magreza e risco de sobrepeso, 68\% (19) apresentou eutrofia, $21 \%$ (6) sobrepeso, $7 \%$ (2) 
obesidade e $1 \%$ (1) obesidade grave, no total de 28 meninas. Quanto a altura nota-se proporções semelhante de adolescentes com altura entre $1,0-1,49 \mathrm{~m} \mathrm{(48,4 \% )}$ e entre 1,5 - 1,99 (51,6\%) ( $p=0,8989)$. Além disso, a maioria dos adolescentes apresentam peso entre 40 a $49 \mathrm{Kg}(40,3 \%)$, enquanto apenas $1,6 \%$ dos estudantes pesam entre 90 a $99 \mathrm{Kg}(\mathrm{p}<0,0001)$ (Figura 2).

Figura 2 - Avaliação de altura dos sujeitos da pesquisa.

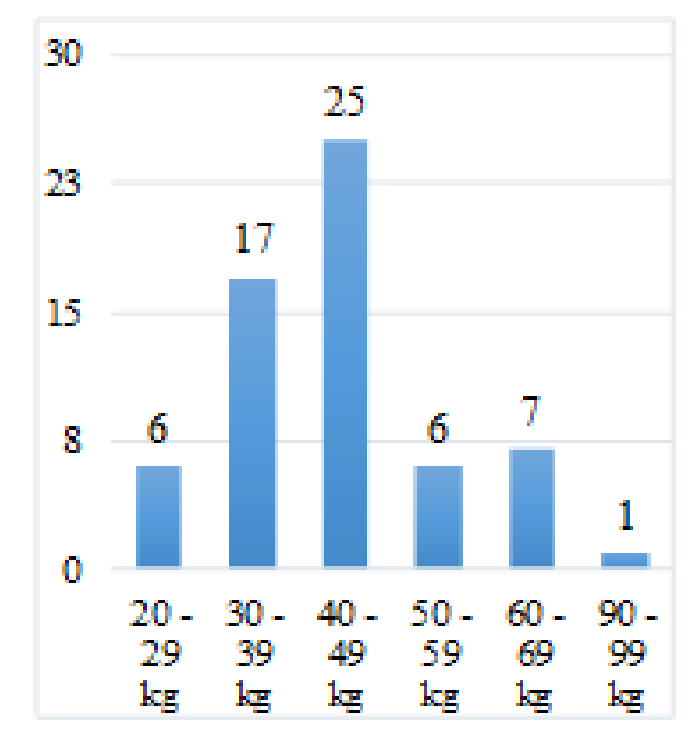

Fonte: Oliveira KBO, et al., 2020.

Em relação a circunferência abdominal, percebe-se uma menor frequência de indivíduos com medida acima de $90 \mathrm{~cm}(3,2 \%)$ e maior frequência entre 60 e $69 \mathrm{~cm}(42,0 \%)(p<0,0001)$ (Figura 3). Esse dado indica ao aumento de gordura abdominal, o qual é um sinal indicador da obesidade e sinalizador de futuras doenças como hipertensão arterial sistêmica e diabetes mellitus.

Figura 3 - Avaliação da circunferência abdominal dos sujeitos da pesquisa.

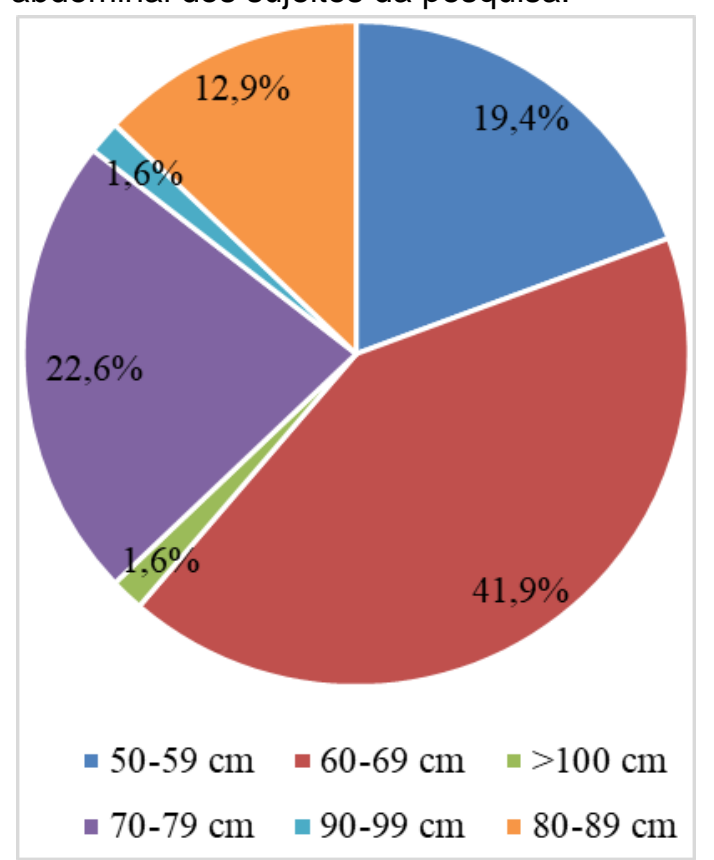

Fonte: Oliveira KBO, et al., 2020. 


\section{DISCUSSÃO}

No presente estudo verificou-se uma proporção de sobrepeso de $16 \%$ para faixas etárias entre 11 a 15 anos, sendo 34 rapazes e 28 moças. $\mathrm{Na}$ análise para os diferentes gêneros, percebeu-se maior frequência de sobrepeso entre as meninas. Ainda nesse contexto, a prevalência de obesidade, para ambos os sexos, foi de $11,2 \%$, havendo prevalência de aproximadamente $11 \%$ de obesidade entre as meninas, com as mesmas idades previamente citadas, e também, $12 \%$ no sexo oposto.

Em comparação, a Pesquisa de Orçamento Familiar (POF) realizada pelo Instituto Brasileiro de Geografia e Estatística (IBGE) nos anos de 2008-2009, revelou que o excesso de peso foi diagnosticado em cerca de um quinto $(20,5 \%)$ dos adolescentes avaliados pelo o Instituto. Além disso, quadros de obesidade corresponderam, nos dois sexos, a cerca de um quarto $(4,9 \%)$ do total de casos de excesso de peso (BRASIL, 2010). Dessa forma, a proporção de obesidade geral da Escola Pública em questão, revela-se acima do esperado quando comparada ao atual quadro do território nacional. Esse achado pode estar relacionado à associação de renda familiar e fatores socioeconômicos com a alimentação.

Em uma análise da prevalência e fatores associados ao sobrepeso e à obesidade em adolescentes, entre 14 e 19 anos, em Pernambuco, a taxa de sobrepeso e obesidade foi de $11,5 \%$ e 2,4\%, respectivamente (ABRANTES MM, et al., 2002). Revela-se assim, dados dos adolescentes do Benguí II opostos à de tal realidade. Além disso, nessa pesquisa não se observou diferença significativa entre a proporção de moças e rapazes classificados como casos de "sobrepeso" e de "obesidade". Essa informação assemelha-se aos dados encontrados pelo atual estudo apenas quanto a proporção de obesidade nos diferentes sexos da escola de Belém, já que se percebeu diferença significativa entre a prevalência de sobrepeso dos meninos (12\%) e das meninas $(21 \%)$.

Em uma pesquisa desenvolvida no Rio de Janeiro, avaliou que 1945 alunos de uma rede municipal de ensino, 10,4\% estavam com sobrepeso e 6,4\% estava em obesidade, comparado com um estudo descrito em Belo Horizonte que a prevalência de obesidade encontrou 2,1\% e outros estudos brasileiros que encontraram uma estatística entre 5,2\% e 11,2\% (ABRANTES MM, et al., 2002). O que confirma mais uma vez, que a frequência de adolescentes com excesso de peso contrasta-se das demais evidências.

Embora amplamente usado e consolidado, o IMC isoladamente não reflete ou classifica de maneira inequívoca os riscos cardiometabólicos, sendo neste caso, um importante fator a ser considerado (avaliado) a deposição de gordura intra-abdominal (HEYDE MEDV, et al., 2000). Portanto, o PC (perímetro da cintura) é uma medida adicional ao IMC em crianças, considerada uma medida de simples avaliação e interpretação, não invasiva e que não requer infraestrutura complexa.

$\mathrm{Na}$ última década, estudos já destacam que para diferentes idades e sexo existem pontos de cortes distintos, utilizados em diferentes países para classificar a adiposidade abdominal em adolescentes. Nesse sentido, determinaram ser o percentil 85 um ponto crítico para identificar o excesso de peso por meio do IMC em adolescentes de ambos os sexos (KATZAMARSKY PT, 2004).

Embasado em tais evidências, nessa pesquisa, a partir do ponto de corte (Percentil $\geq 85$ ) verificou-se maior frequência de adolescentes com circunferência abdominal entre 80 a 89 centímetros e menor frequência para os que possuem perímetro maior que 100 centímetros (apenas um indivíduo). Dentre os de 80 a $89 \mathrm{~cm}$, existem indivíduos tanto com sobrepeso quanto com obesidade, em contrapartida, a pessoa com circunferência maior que $100 \mathrm{~cm}$, foi diagnostica com obesidade grave. Adolescentes com percentil $\geq$ 85 representam $27,4 \%$ do total da amostra.

Os resultados encontrados no presente estudo representam o dobro dos encontrados em escolas da rede pública no Rio Grande do Sul que avaliou adolescentes com idades entre 12 e 17 anos e obteve 13,39 $\%$ da amostra acima do percentil 85 (FERNANDES RA, et al., 2009).

No entanto, em comparação aos encontrados por que avaliaram escolares da cidade de Maringá com idades de 12 a 18 anos, a pesquisa aproximou-se do valor de prevalência descoberto (33 \%) (MALTA MC, et al., 2014). 


\section{CONCLUSÃO}

A avaliação dos resultados mostrou que a prevalência de sobrepeso entre o grupo feminino é maior que o masculino. Além disso, a obesidade em ambos os sexos se mostrou acima do esperado, fazendo então com que o número de adolescentes obesos seja maior que o esperado, comparando-se com o quadro que se vê em território nacional. Foi possível avaliar que o atual estudo contribuiu para encontrar o perfil dos estudantes da escola que pode servir de base para o desenvolvimento de ações em saúde direcionadas ao público. Já a principal limitação deste estudo foi de pais e/ou responsáveis não demonstrarem interesse para o estudo não assinando o termo desenvolvido pelos autores o que prejudicou de certa forma até a educação em saúde dos indivíduos da pesquisa que poderiam se beneficiar com o estudo. Dessa forma, ainda há a necessidade de mais estudos nesta área a fim de desenvolver e construir mais medidas preventivas e/ou terapêuticas mais eficientes para esta problemática.

\section{REFERÊNCIAS}

1. ABESO. Diretrizes Brasileiras de Obesidade. 3. ed. São Paulo. 2009.

2. ABRANTES MM, et al. Prevalência de sobrepeso e obesidade em crianças e adolescentes das regiões Sudeste e Nordeste. Jornal Pediatria, 2002, 78(4): 335-40.

3. BRASIL. Ministério da Saúde. Pesquisa de Orçamentos Familiares. BRASIL. 2010.

4. BRASIL LMP, et al. Excesso de peso de escolares em região do Nordeste Brasileiro: contraste entre as redes de ensino pública e privada. Revista Brasileira de Saúde Materno Infantil. 2007; 7(4): 405-412.

5. BRASIL. Ministério da Saúde. SISVAN Municipal - Estado Nutricional dos Usuários da Atenção Básica.

6. BRASIL. Ministério da Saúde. Orientações para a coleta e análise de dados antropométricos em serviço de saúde. 2011.

7. BRASIL. Vigilância de Fatores de Risco e Proteção para Doenças Crônicas por Inquérito Telefônico. 2014.

8. BRASIL. IBGE. Pesquisa de Orçamentos Familiares 2008-2009. Rio de Janeiro, 2010.

9. CAMPOS LA, et al. Prevalência de sobrepeso e obesidade em adolescentes escolares do município de Fortaleza, Brasil. Revista Brasileira de Saúde Materno Infantil, 2007; 7(2): 10-20.

10. DUMITH SC, et al. Sobrepeso e obesidade em crianças e adolescentes: comparação de três critérios de classificação baseados no índice de massa corporal. Revista Panamericana de Salud Pública, 2010, 28(1): 30-35.

11. FERNANDES RA, et al. Proposta de Pontos de Corte para indicação da obesidade Abdominal entre Adolescentes. Arq Bras Cardiol 2009; 93(6): 603-609.

12. GOLDMAN L, et al. Cecil - Medicina. 23. ed. Tradução: Adriana Pittella Sudré. Rio de Janeiro: Elsevier Editora Ltda.,2011.

13. GUYTON AC, et al. Tratado de Fisiologia Médica. 12. ed. Tradução Alcides Marinho Junior. Rio de Janeiro. Elsevier Editora Ltda. 2011.

14. HEYDE MEDV, et al. Perfil nutricional de adolescentes da cidade de Curitiba. Anais do Simpósio: Obesidade e anemia carencial na adolescência. Instituto Danone; 2000 jun 8-9; Salvador, BA. Salvador; 2000: 227.

15. JUNIOR JGS. Prevalência de sobrepeso e obesidade em escolares da cidade de Veríssimo-MG. In: Coleção Pesquisa em Educação Fìsica. 2012, 11(3): 212-217.

16. KATZMARZYK PT. Waist circumference percentiles for Canadian youth 11-18y of age. Eur J Clin Nutri, 2004; 58(7): 1011-5.

17. MALTA MC, et al. Evolução anual da prevalência de excesso de peso e obesidade em adultos nas capitais dos 26 estados brasileiros e no Distrito Federal entre 2006 e 2012. Rev Bras Epidemiologia; 2014; Suple: 267-276.

18. RIBEIRO RQC, et al. Prevalência da obesidade em escolares adolescentes na cidade de Belo Horizonte. Resultados parciais do II Estudo Epidemiológico. Anais do Simpósio: Obesidade e anemia carencial na adolescência. Instituto Danone; 2000 jun 8-9; Salvador, BA. Salvador; 2000: 237-38

19. SBP. Obesidade na infância e adolescêcia: Manual de Orientação. 2. ed. Rio de Janeiro, 2012. 